\title{
Some syntactic determinants of sentential complexity'
}

The perceptual complexity of three lists of self-embedded sentences was evaluated in terms of the accuracy and time required for their paraphrase. The lists differed by the presence of relative pronouns in one list, their absence in a second and by the addition of adjectives to the third. It was predicted that the presence of the relative pronouns would effect the only significant change in performance. In both auditory and visual presentations of the sentence lists, the presence of the relative pronouns proved to be facilitating, while the presence of the adjectives produced no significant changes.

A number of early psycholinguistic studies of generative grammar appear to have been motivated by the hypothesis that, insofar as sentential complexity is a function of syntactic variables, the complexity of a sentence is measured by the number of grammatical rules employed in its derivation. We shall refer to this as the Derivational Theory of Complexity (DTC).

DTC can be made explicit in the following way. Consider a generative grammar $G$ of the language $L$ and a sentence $S$ in the range of $G$. It is possible, in principle, to define a metric which for every pair $\left(G_{i}, S_{i}\right)$ specifies the number $N_{i}$ of rules (or elementary operations, or whatever) $G_{1}$ requires to generate $S_{j}$. DTC in its strongest form is the claim that the size of $N_{i}$ is an index of the complexity of $S_{i}$. In particular, two sentences assigned the same number are equally complex, and, of two sentences assigned different numbers, the larger number is assigned to the more complex sentence.

The literature on DTC and related theories has been reviewed elsewhere (Fodor \& Garrett, 1966). We have argued that the theoretical justification for DTC is questionable and that its success in predicting experimental results has been only indifferent. In the present paper we offer a different approach to understanding the role of grammatical variables in sentential complexity, together with some experimental results which appear to militate in its favor.

Understanding a sentence requires recovering the grammatical relations that obtain among its parts. For example, anyone who understands (1) must observe that "John" is the subject of the sentence, that the sentence contains a predicate adjective "eager," and so on.

(1) John is eager to please

Sometimes, however, the fundamental relations instanced by a sentence are represented only in a very distorted way by the arrangement of phrases, inflections, etc., that the sentence exhibits. For example, though the syntactic relations in (2) appear to be

\section{(2) John is easy to please}

the same as those in (1), it is evident that this appearance must be misleading. To understand (2), one must understand that "John" is really the object of the sentence; i.e., that the sentence is a distorted version of (3) in a way that (1) is not a distorted version of (4).

(3) It is easy for someone to please John

(4) It is eager for someone to please John

To capture the fact that the real grammatical relations in a sentence may be quite abstractly represented by its apparent grammatical relations, linguists postulate a "deep structure" in which the real relations are explicitly marked (cf., Chomsky, 1965; Katz \& Postal, 1964; et al). Thus, while the apparent (surface) structures of (1) and (2) are identical, their deep structures differ. The deep structure of (2), but not that of (1), contains a structure from which (3) can be derived.

We may now understand why some theorists have adopted accounts of complexity like DTC. The problem of understanding a sentence can be conceptualized as requiring the recovery of the deep structure corresponding to a given surface structure. DTC asserts that, ceteris paribus, the more rules required to convert the deep structure of a sentence into its surface structure, the more complex the sentence must be.

It must be noticed, however, that accepting the transformational view of grammar does not require accepting DTC, nor would the confirmation of DTC be in any sense a confirmation of the grammar. Rather it is far from obvious that one ought to expect a correspondence between the complexity of the grammatical operation of converting a base structure into a surface structure and the complexity of the perceptual operation of converting a surface structure into a base structure. Though it is widely accepted that grammars are theories neither of speakers nor of hearers, much past work in psycholinguistics suggests a failure to appreciate the full implications of this fact; namely that the grammar constrains only the output of the perceptual model (i.e., the latter must recognize the set of structural descriptions 
generated by the former). In particular, the grammar in no way imposes constraints either on the set of facts about the input that the perceptual model may exploit in computing structural descriptions, or upon the operations it runs through in the course of the computation.

We suggest that the complexity of a sentence is a function not (or not only) of the transformational distance from its base structure to its surface structure but also of the degree to which the arrangement of elements in the surface structure provides clues to the relations of elements in the deep structure. To a certain extent this hypothesis about complexity and DTC yield convergent experimental predictions. This is because increasing the distance from base to surface structure tends, on the whole, to obliterate surface structure clues to deep structure. In general, transformations deform the base structure trees that they apply to. Nevertheless, insofar as increasing the number of transformations tends to Increase complexty, we suggest that this is not because of the increased transformational distance between base and surface structure per se but rather because of the consequent obliteration of the surface structure clues upon which the reconstruction of deep structure depends. We shall see in the discussion that profound theoretical questions about the relation of a generative grammar to a psychological model of speech perception hang upon this distinction.

Moreover, DTC and the present proposal differ in that the former appears to require us to make counter-intuitive statements about complexity in certain important cases. For example, since phrases like (5)

(5) The red house

are transformationally derived from phrases like (6)

(6) The house which is red

DTC appears to require that (5) be more complex than (6). Similarly it requires that short passives like (7) be more complex than the long passives, like (8), from which they are transformationally derived.

(7) The boy was hit

(8) The boy was hit by someone

These are not isolated cases. Rather they llluminate a general difficulty with DTC. Sentences which are transformationally "close" to their deep structure sources are generally more richly grammatically elaborated than sentences which are transformationally distant from their deep structures. This is because, as we have seen, transformations tend to destroy structure. Notice, however, that this means that whereever one sentence lies in the derivational history of another, DTC is required to predict that the more fully elaborated sentence is perceptually simpler than its less elaborated counterpart. Hence, in all such cases DTC is committed to counter-intuitive predictions.

\section{EXPERIMENTS}

The experiments to be described here turn upon the fact that certain lexical items, which appear as the result of embedding transformations, are optionally deletable. For example, the "self-embedded" sentences (9) and (10) are fully grammatical and fully synonymous. In (9), however, the word whom, which results from the embedding of the dog bit the man in the man died, has been deleted.

(9) The man the dog bit died

(10) The man whom the dog bit died

It is hypothesized that the presence of the relative pronoun in sentences like (10) should make them perceptually less complex than corresponding sentences like (9). This is because the relative pronoun provides a surface structure clue to semantically cruclal deep structure relationships. Specifically it is evidence of the application of an embedding transformation, and that transformation can apply only where certain grammatical relations hold between the noun phrases (NPs) in the sentence.

In particular, for the lexical material we employed, the surface structure constellation $\mathrm{NP}_{1}$ relative pronoun, $\mathrm{NP}_{2}$ can appear grammatically only where $\mathrm{NP}_{2}$ is the base structure subject of a transitive verb of which $\mathrm{NP}_{1}$ is the base structure object. Thus the relative pronoun in a sentence like (10) provides an immediate clue to the deep structure of the sentence. This clue is absent in sentences like (9), where the initial constellation of NPs is compatible, e. g., with the list structure displayed in (11).

(11) The man the dog and the girl went swimming

The restriction of this analysis to sentences containing lexical material of the type we have employed is essential. There exist types of verbs that can follow the constellation $\mathrm{NP}_{1}$ rel $\mathrm{NP}_{2}$ in sentences where the NPs do not exhlbit the sorts of subject/ object relations exemplified by (9) and (10). For example, verbs that take complements, as in (12); middle verbs, as in (13); and verbs that take indirect objects, as in (14). On the other hand, an heuristic

(12) The boy that the man wanted Joan to meet was ill

(13) The amount that the book cost was excessive

(14) The girl that the boy gave the book to was pretty

based on the presence of the relative pronoun in the sequence $\mathrm{NP}_{1}$ rel $\mathrm{NP}_{2}$ will work without exception for sentences containing only simple transitive verbs. In short, we must assume that, if the heuristic (i.e., "Given the sequence $\mathrm{NP}_{1}$ rel $\mathrm{NP}_{2}$ assume the $\mathrm{NPs}$ are related to each other as object and subject respectively of the same verb." ) is to be useful to Ss, 
it must interact with their knowledge of the character of the verb(s) in the stimulus sentences. An assumption of the present approach is, thus, that $S$ must have available a richly structured syntactic analysis of the lexical items of his language. In particular, the lexical items are presumed to be classified in terms of the deep structure configurations they can enter. As a consequence of this assumption, it is predicted that the errors $S$ makes on tasks involving comprehension of sentences with sequences like $\mathrm{NP}_{1}$ rel $\mathrm{NP}_{2}$ will reflect his knowledge of the deep structure types the lexical material in such sentences permits. We will return to these and related considerations in the discussion.

Self-embedded sentences like (9) and (10) above thus exhibit features that are relevant to testing the significance of certain types of surface structure clues to deep structure configurations. We have employed them in the present experiments because, with iteration of the self-embedding operation, Ss have great difficulty in understanding them. ${ }^{2}$ This provides an opportunity for the presumed facilitatory effects of surface structure clues to be revealed more strongly than in the case of sentences which Ss find easy to understand.

In the first experiment described below, the presence of the presumed facilitatory effect of the presence of relative pronouns is demonstrated. Subsequent experiments deal with possible alternative explanations of this effect.

\section{Experiment 1}

Nine pairs of sentences with two embeddings each were constructed. The list below gives the sentences with the optionally deletable items in parentheses.

(1) The pen (which) the author (whom) the editor liked used was new.

(2) The tiger (which) the lion (that) the gorilla chased killed was ferocious.

(3) The boats (which) the rocks (that) the waves covered sank were large.

(4) The cigarette (which) the match (that) the flame ignited lit smoked.

(5) The car (which) the man (whom) the dog bit drove crashed.

(6) The window (which) the ball (that) the boy threw hit broke.

(7) The bicycle (which) the boy (whom) the policeman stopped turned was lost.

(8) The shot (which) the soldier (that) the mosquito bit fired missed.

(9) The man (whom) the girl (that) my friend married knew died.

Two groups of sentences are derivable from this list. The group in which the relative pronouns were deleted will be referred to as Group I; the group in which the relative pronouns are retained will be referred to as Group II. Sentence Groups I and II were tape recorded for presentation to Ss. In the materials for Experiment 1, the sentences were read in what $E$ judged to be a "neutral" manner; that is, the intonation contour was kept flat and expressive devices like emphatic stress, long pause, etc., were avoided as far as possible.

Twenty Ss were randomly assigned to each of two groups of 10. One $S$ group heard sentence Group I and the other heard sentence Group II. Ss were run individually; the material was presented binaurally through earphones in a quiet environment.

Ss were all MIT undergraduates paid for their voluntary participation. They were instructed to restate each sentence in their own words immediately following its presentation. Ss were not allowed a rote repetition of the sentence. This was because it was found in preliminary work that Ss could produce rote repetitions of sentences of the type used here even when they professed not to understand the sentences and could not paraphrase them. Clearly, the present hypothesis is relevant only in cases where the material is treated as a sentence, not merely as a list for rote recall. Ss had five successive attempts at each sentence. Responses were tape recorded.

Two basic scores were computed. The first was the interval between the end of the presentation of the stimulus sentence and the onset of S's vocalization. We will refer to this interval as the "response delay." Second, S's paraphrase was scored to determine his grasp of the subject-object relations among the NPs. The results will be reported here, however, in terms of the grammatical relations scores weighted by the response delay scores. That is, the value reported for each sentence will be the mean number of correct subject-object relations reported per trial, divided by the median response delay. This value may be thought of as number of grammatical relations correctly recovered per sec. of response delay (it must be borne in mind, however, that the values were determined from group means). Here and subsequently the pattern of results for both the response delay measure and grammatical relations measure is substantially the same. Where there is any difference between the combined score and either of the two independent measures, it will be noted. However, the combined score is presumably the most appropriate measure, since facilitation may be exhibited either in decreased response delay or in increased accuracy of paraphrase-or both.

Results. Table 1 presents the combined score measures for each of eight stimulus sentences. 3 The difference between Groups I and II is significant for $\alpha=.05$ (Wilcoxon matched-pairs, signed ranks test, one tail). All elght sentences showed larger combined scores for the group with the relative pronouns present. In fact, the presence of the relatives made the sentences easier to understand whether one takes 
Table 1

Mean number of subject-object relationships recovered per second of response delay

\begin{tabular}{lcccccc} 
& Group I Group II & & Group I Group II \\
\hline 1. The pen & .14 & .49 & & 5. The car & .66 & .88 \\
2. The tiger & .15 & .47 & & 6. The ball & .80 & 1.00 \\
3. The boot & .29 & .48 & & 8. The shot & .51 & .99 \\
4. The cigarette & .18 & .57 & & 9. The man & .24 & .38 \\
\hline
\end{tabular}

the response delay or the accuracy of information retrieval as the criterton. Seven of the eight sentence pairs showed response delay differences in the predicted direction. The one reversal is by far the smallest of the differences. (This sentence is also one of a subgroup of three in which an additional source of variation is operative, i.e., semantic selectional restriction between nouns and verbs. This will be discussed below.) All elght sentences show the greater number of grammatical relations correctly reported for the versions in which relatives were present.

There are four important alternative explanations of the differences between Groups I and II which must be considered. First, one might suppose that inadvertent changes in the prosodic features were introduced during the reading of the sentences in Group II. That is, one might suppose that it is not the relative pronouns per se that produced the facilitation in Experiment 1, but rather that their introduction affected the reading of the stimulus material (i.e., a more expressive reading was given for Group II than for Group I). It is clear that the prosodic features are capable of conveying some of the sorts of information that we have hypothesized is provided by the presence of the relative pronouns. Hence, though in the judgment of the $\mathrm{Es}$ the readings for both groups in Experiment 1 were comparably expressionless, the possibility of inadvertent prosodic clues must be considered.

Second, it might be argued that the presence of the relatives somehow provides a cue to the segmentation of the sentence. That 18 , it could be claimed that the difficulty with these sentences arises not out of any differential availability of the grammatical relationships but in the preprocessing required to establish segmentation of the utterance. The difficulty with the deleted relative sentences would thus be attributed to the S's uncertainty about which objects in the sentence to treat as units in its analysis rather than to his inability to grasp the grammatlcal relations which obtain among them.

The third alternative is that since the relatives are ordinarily redundant (the information they provide is carried positionally and by the prosodic features), their presence thus reduces the rate of presentation of information and gives $S$ more time to retrieve the grammatical facts about the sentence.

The fourth alternative is simply DTC, referred to earlier. That 1s, it might be assumed that the addition of the relative pronoun deletion transformation
Table 2

Mean number of subject-object relationships recovered per second of response delay

\begin{tabular}{lccllll} 
& Group I Group II & & \multicolumn{1}{c}{ Group I Group II } \\
\hline 1. The pen & .31 & .78 & & 5. The car & .71 & .86 \\
2. The tiger & .36 & .39 & 6. The ball & .70 & .80 \\
3. The boat & .51 & .48 & 8. The shot & .68 & .76 \\
4. The cigarette & .45 & .71 & 9. The man & .29 & .36 \\
\hline
\end{tabular}

to the derivational history of the sentences in Group II makes them more complex than the sentences of Group I.

Four variations of Experiment 1 were run in order to evaluate these alternatives. In order to determine the relevance of prosodic cues to the facilitation effects found in Experiment 1, such cues were intentionally introduced in Experiment 2. In Experiment 3, rate of presentation is made slower for sentences in Group I. In Experiment 4, the relevance of the serial input and fixed rate of information presentation required by the auditory mode is evaluated through a visual analogue of the experiment. In Experiment 5, the length of the derivational history for sentences in Group I is increased by several transformations.

\section{Experiment 2}

All the stimulus materials and conditions of presentation of sentences were as in Experiment 1. The sentences were re-recorded, however. Instead of a neutral reading as in Experiment 1 , an effort was made to read the sentences expressively. If the prosody is capable of doing the work of the relatives, we should expect to eliminate any differences between Groups I and II not attributable to other factors (as slower rate of presentation).

Results. Table 2 reports the combined scores for the eight sentences. The difference between the two groups is significant for $\alpha=.05$. (If response delay and grammatical relations measures are considered separately, we find both in the predicted direction. The difference between the two groups is significant for response delay but not for the grammatical relations measure.) If one compares performance in these groups with that of the groups in Experiment 1 , it is clear that the prosodic cues improved scores significantly in the relatives deleted group. In spite of this reduction, the differences between the relatives deleted and the relatives present groups remains. Further, Group II (relatives present) without prosodic cues (1.e., Group II in Experiment 1) is significantly superior $(\mathrm{p}<.01)$ to Group I (relatives deleted) with prosodic cues (i.e., Group I in Experiment 2). Evi-

Table 3

Mean number of subject-object relationships recovered per second of response delay

\begin{tabular}{llll}
\hline 1. The pen & .21 & 5. The car & .56 \\
2. The tiger & .20 & 6. The ball & .55 \\
3. The boat & .30 & 8. The shot & .59 \\
4. The cigarette & .25 & 7. The men & .26 \\
\hline
\end{tabular}


Table 4

Mean number of subject-object relationships recovered per second of response delay

Visual Visual
Group I Group

Visual Visual Group I Group II

\begin{tabular}{lrrrrrr}
\hline 1. The pen & .35 & .48 & & 5 . The car & .81 & 1.17 \\
2. The tiger & .25 & .29 & & 6 . The ball & 1.19 & 1.13 \\
3. The boat & .23 & .43 & 8. The shot & 1.00 & 1.25 \\
4. The cigarette & .33 & .64 & & 9. The man & .30 & .43 \\
\hline
\end{tabular}

dently the prosodic features are helpful but they cannot account for the difference between Group I and Group II observed in Experiment 1.

\section{Experiment 3}

A copy of the tape containing stimulus sentences for Group I in Experiment 1 was made. At the points in the sentence where relatives occur in Group II, a piece of blank tape of appropriate length was spliced into the sentence (mean values for the relatives' durations; $330 \mathrm{msec}$. for the first relative position and $260 \mathrm{msec}$. for the second relative position). This made the durations of the sentences in Group I approximately equal to those in Group II. All other conditions were as in Experiment 1.

Results. Table 3 presents the values on the combined measure for the eight stimulus sentences. When compared with the values for Group I (see Table 1) we see that there is no significant improvement in the scores produced by the reduced rate of presentation of the sentences.

\section{Experiment 4}

As an additional control on the possible effects of rate of presentation, a visual version of the experiment was performed. The sentences were typed separately on $3 \times 5$ in. file cards in capital letters. Ss were presented with each card for a period of $3 \mathrm{sec}$. The card was then removed and Ss were required to respond as they were in the other experiments. All other conditions were as in Experiment 1.

Results. Table 4 presents the values on the combined score for sentence Groups I and II in visual presentation. Just as in the auditory presentation, the version in which the relative pronouns are present is significantly better than the group in which they are absent. Since in this version of the experiment the rate of presentation of information is presumably equivalent for the two groups, the significant differences in Ss' performance evidently cannot be attributed to a greater available processing time for Group II. Nor can it be attributed to a segmentation problem

Table 5

Mean number of subject-object relationships recovered per second of response delay

\begin{tabular}{llll}
\hline 1. The pen & .42 & 5. The car & .62 \\
2. The tiger & .21 & 6. The ball & .82 \\
3. The boat & .48 & 8. The shot & .84 \\
4. The cigarette & .21 & 9. The mon & .20 \\
\hline
\end{tabular}

peculiar to the auditory mode.

\section{Experiment 5}

In this experiment, the If .2gth of derivational history of the sentences in Group I was greatly increased by the addition of an adjective before each of the first two Noun Phrases. Once again, sentences were read in a neutral intonation to permit comparison with Group I in Experiment 1.

To understand the nature of this control, we must consider the syntactic analysis of adjective-noun sequences. Consider, in particular, the sequence "tired soldier" occurring in the sentence: "The first shot the tired soldier the mosquito bit fired missed." The underlying structure is the configuration (The soldier rel fired the shot) where rel dominates (the soldier is tired) (see Fig. 1). The order of operations in the derivation is roughly the following. Relativization applies to produce (the soldier who is tired). Who is deletes optionally to yield the intermediate form (the soldier tired) which by mandatory permutation of the adjective with the noun yields the tired soldier.

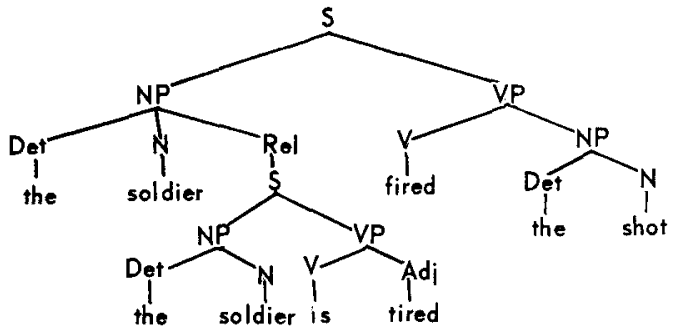

Fig. 1. Schematic deep structure tree for the sentence: the tired soldier fired the shot.

In short, each of the two added adjectives introduces three transformations, so that each adjective control sentence has six more transformations in its derivational history than the comparable Group I version and eight more than the comparable Group II version. If, then, increase in the length of the derivational history by two deletion operations is presumed to account for the difference in complexity between the relative and deleted relative version of the stimulus sentences, we would expect that the increase of length by an additional six operations ought to make S's performance on the adjective versions far the worst in our study.

Results. The comparison of the adjective and deleted relatives groups on the weighted grammatical relations measure is given in Table 5. Clearly, the addition of the adjectives fails to produce a performance decrement.

On the contrary, the adjective group is better than the deleted relatives group $(p<.13)$. This, of course, is just the reverse of the DTC prediction. In order to determine whether this result was 
consistent we reran the adjective materials with visual presentation. The results were that performance was slightly but not significantly $(p<.20)$ worse than for the visual presentation of Group I (cf., Experiment 4). In neither the auditory nor the visual presentations were the differences between Group I values and values for the adjective versions of the stimulus sentences as great as those produced by the presence of the relative pronouns. The mean changes across sentences effected by the presence of relative pronouns was .29 for auditory presentation and .17 for visual presentation. The changes effected by the adjectives in Experiment 5 were an improvement of .10 for auditory presentation and a reduction of .02 for visual presentation.

It is possible that the improved performance for the auditory presentation of the adjective sentences is attributable to additional prosodic cues in that group. As Experiment 2 showed, performance on this task can be significantly improved by such cues. In any event, it seems clear from the two experiments with the adjective versions of the stimulus sentences that the drastic performance decrement predicted by DTC is not forthcoming.

\section{GENERAL DISCUSSION}

A convenient overview of the several experiments can be obtained by relating the experimental groups to each other on each of the stimulus sentences. Using the combined measure (grammatical relations scores weighted by response delays), the scores for each auditory condition can be ranked for each sentence-as, for example, on Sentence 1 the groups rank as follows (lowest scores to highest):

\begin{tabular}{lr}
\multicolumn{2}{c}{ Sentence number 1} \\
Group I & No intonation \\
Group I & Blank tape \\
Group I & With intonation \\
Group I & With adjectives \\
Group II & No intonation \\
Group II & With intonation
\end{tabular}

If one does similarly for the remaining sentences and then sums the ranks for each condition, an ordering of the experimental groups is determined as follows:

$\begin{array}{lr}\text { EXPERIMENTAL CONDITION } & \text { SUM OF RANKS } \\ \text { No Intonation, no rels } & 13.5 \\ \text { Blank tape } & 16.0 \\ \text { Auditory adjectives } & 26.0 \\ \text { Intonation, no rels } & 30.0 \\ \text { Intonation, rels } & 38.5 \\ \text { No intonation, rels } & 44.0\end{array}$

No pair of adjacent groups in the above array are significantly different except where the relatives are introduced. More strikingly, there is no significant difference between the groups with relatives, even though the corresponding difference between the deleted relatives groups is significant. That is, though the addition of prosodic cues produces significant increases in scores for the deleted relatives groups, this is not the case for groups where relatives were present. In short, in the presence of the relative pronouns apparently the other facilitating variables have little effect.

In the analysis of the results above, the grammatical relations score used referred only to S's performance on subject-object relations among the NPs. Further analysis reveals facilitation for relations between nouns and verbs as well, e.g., S's recovery of the relationship between $N P_{1}$ and $V P_{3}$ is facilitated. However, the facilitation for such relations was found to be significant in Experiment 1 but not in Experiment 2. That is, it appears that the facilitation effect of the relative pronouns extended to relations other than subject-object when there were few prosodic cues. When the prosodic cues were provided, however, S's performance on the two groups of sentences is strongly discriminated only along the lines of the heuristic device we have suggested the relative pronoun permits.

Finally, in evaluating the effect of the rels, Sentences 5, 6, and 8 merit special consideration. In these sentences the nouns and verbs were chosen such that certain of their combinations are either grammatically impossible or intuitively absurd. Thus in Sentence 5 , analyses like the car bit or the dog drove are presumably ruled out a priori. Schlesinger (1966) has shown that such restrictions enhance S's performance on self-embedded constructions, presumably by narrowing the combinatorial possibilities $S$ has to consider. In the present data, too, the occurrence of such restrictions clearly facilitates the S's performance. It should be noted, however, that even on these sentences the presence of the relatives significantly improves S's performance.

If one assumes that the effect of retaining the relative pronouns is clearly established by these results, it remains to consider what implication these results have for a theory of how the syntactic structure of sentences is understood.

The most important consequence of the present results is not specific to the perception of selfembedded constructions. Rather, it concerns the relation between the grammatical operations (of transformation, etc.) employed by a syntax in the generation of representations of sentences and the mental operations that must be presumed on the part of the hearer who understands a sentence.

On theories like DTC, this relation is assumed to be very close indeed. One predicts a correlation between the length of the derivational history of a sentence and its perceptual complexity for one of two reasons. Either one assumes that in understanding a sentence the hearer literally runs through the set of operations a grammar employs in gen- 
erating the sentence, or at least one assumes that for each grammatical operation there is a corresponding decoding operation.

Such views have their most explicit realization in the "analysis by synthesis" model of speech perception. In that model the grammar is employed as a source of internally generated sentential analyses. These analyses are compared with some representation of the input signal to be recognized. The comparison procedure halts when a match is effected between the internally generated signal and the input (cf., Halle \& Stevens, 1964; Matthews, 1962).

The interest of such models, for present purposes, is that they explicitly assume that the process of grammatically generating a sentence is part of the process of recognizing it. This means that such models claim psychological reality not only for the grammatical relations marked by a structural description but also for the grammatical operations employed in generating the description.

Clearly, on the analysis by synthesis model or any other model which assumes that the operations of the grammar are isomorphic to some subset of the operations of the recognition device, DTC ought to be true. The present results therefore suggest that such models should be viewed with some suspicion. There are two relevant points. On the one hand, the results with the adjectives suggest that there are at least some cases where lengthening of the derivational history of a sentence falls to produce a significant increase in its perceptual complexity. On the other hand, the demonstrable facilitation introduced by the relative pronouns suggests alternatives to grammar-isomorphic recognition procedures.

In short, it appears that $S$ has avallable heuristics for making fairly direct inductions of base structure configurations (i.e., of fundamental grammatical relations) given relevant surface structure information. These heuristics employ information represented in the grammar, but they are not themselves grammatical rules in the usual sense of rules used to generate sentences. The present results suggest some insights into what the nature of these heuristics might be.

We have seen that it is a precondition of facilltation by the relative pronoun that $S$ has and employs an analysis of the verbs in terms of the base structure configurations into which they can enter. Further support for this view derives from analysis of the erroneous responses in the two cases where our sentences contained verbs which can take deep structures other $\mathrm{NP}_{1} \mathrm{~V} \mathrm{NP}_{2}$; these are "like" (compare Sentence 1 with "The pen the editor liked the author to use...") and "know" (compare Sentence 9 with "The man my friend knew married the girl..."). In both these cases, but in only these cases, S's produced errors compatible with deep structure analyses other than $N P_{1} \quad N P_{2}$; that is, all other errors preserve the essentlal subject-verb-object base structure configuration.
These considerations suggest what we take to be important possibilities for further research. It seems likely to us that the program Ss use to recover the grammatical structure of sentences has two primary components: on the one hand, it must consult a lexicon which classifies the verb in the sentence according to the base structure configurations it may enter. Second, It must run through each such deep structure configuration, asking whether the surface material in the sentence can be analyzed as a transformed version of that deep structure. Thus, for example, in analyzing a Bentence like "John expected Mary to leave," we assume that $S$ must ask which of the deep structure configurations possible for "expect" the sentence exemplifies. In particular, we assume that he knows that "expect" can take for/to or that complementation or a direct object, and we assume that he has and applies tests for each of the various base structure configurations the verb may take. It is at this stage that surface structure features analogous to the presence of the relative pronoun in self-embedded constructions presumably play their role. Thus, it is self-evident that the analysis of the present example as for/to complementation must be facilitated by the presence of the "to" in the surface structure of the sentence.

The action of the surface structure markers in facllitating the recognition of such deep structure features as complement type can sometimes be quite dramatic. For example, that complements are the only ones within which tense can appear in English ("He thinks that John will be late" but "He believes John to be an Idiot" and not "He believes John to will be an (diot"). For this reason, the presence of marked tense is an extremely efficient clue to that complementation in reduced sentences (i.e., in sentences where the word "that" has been deleted). Notice that "He felt the child trembled" is heard as a reduced form of "He felt that the child trembled" and as unrelated to the (tenseless) "He felt the child tremble." Correspondingly, "He felt the children tremble" is ambiguous between the two versions, depending on whether "tremble" is heard as tensed. In these cases, it seems perfectly evident that an effectlve clue to the analysis of the complement is being given by the presence or absence of tense in the surface structure.

Notice that if these examples are typical of the sentence recognition strategies Ss employ, it seems their search routine need not require frequent analysis by synthesis loops through the grammatical rules. Rather the types of structures to be examined in any given case would be drastically constrained if only by the S's information about the deep structure capacities of the verb.

Clearly, this is no more than a sketch of the way syntax recognition might work: on the one hand, there will be cases where applying the surface structure tests for appropriateness of a putative base structure 
analysis requires the restoration of transformationally deleted material, and it is not at all obvious precisely how this is to be accomplished. Second, it is a pressing experimental problem to provide direct and conclusive evidence for the centrality of the lexical analysis (in particular of the verb) in the perceptual process. Nevertheless, if this sort of account is at all plausible, it is self-evident that there would be no theoretical motivation whatever for theories of complexity analogous to DTC. Since the hypothesized relationship between grammatical rules and perceptual heuristics is extremely abstract, this model provides no basis for a notion of perceptual complexity defined in terms of the former.

The most profound problem in psycholinguistics is perhaps to specify the nature of the relation between the grammar and the recognition routine. We have seen that the only a priori requirement upon that relation is simply that the recognition routine must recover the structural descriptions output by the grammar. We may now provide a suggestion for a more informative account: Given the grammar it should be possible to construct, first, a classification of the formatives by reference to their base structure properties; and second, an analysis of the ways in which specified surface structure features represent deformations of base structure configurations. Very roughly, one would expect the first part of this construction to exploit information provided by the lexicon of the grammar and the second part to exploit information provided by the transformations. The fundamental theoretical problem is, then, to characterize the algorithm that performs this construction.

\section{References}

Chomsky, N. Aspects of the theory of syntax. Cambridge: M. I. T. Press, 1965.

Fodor, J., \& Garrett, M. Some reflections on competence and performance. In J. Lyons and R. J. Wales (Eds.), Psycholinguistics papers. Edinburgh: University of Edinburgh Press, 1966.

Halle, M., \& Stevens, K. Speech recognition, a model and a program for research. In J. Fodor and J. Katz (Eds.), The structure of language. New Jersey: Prentice Hall, 1964.

Katz, J., \& Postal, P. An integrated theory of linguistic descriptions. Cambridge: M. I. T. Press, 1964.
Matthews, G. H. Analysis by synthesis of sentences in natural languages. In Proceedings of International Congress on Machine Translation and Applied Language Analysis. London: H.M.S.O. 1962.

Schlesinger, I. M. Sentence structure and the reading process. Unpublished doctoral dissertation, The Hebrew University of Jerusalem, 1966.

\section{Notes}

1. This research was supported in part by a grant from the United States Air Force, (Contract number AF 19(628)-5705). Dr. Garrett also holds an appointment under NIH Training Grant number 5 T1 HD 111-02. We would also like to thank Mrs. Kathryn Tidrick for her work in collection and analysis of the experimental data. 2. The rather puzzling difference between the difficulty of selfembedded sentences with a single level of self-embedding and those with two levels can be understood if we bear in mind that the task of understanding such sentences requires more than the assignment of a subject-noun to each verb. In fact, the sentence must be analyzed into subject-verb-object triples with some nouns appearing as subject of one verb and object of another. That is, it is not just another noun-verb pairing that must be recovered when a second embedding is added, but rather an additional subject-verbobject triple. The interaction of the added elements with those already present results in a striking expansion of the possible analyses of the sentence.

Given one embedding, two nouns must be assigned to each of two verbs as subject and two nouns must be assigned to each of two verbs as object. Hence, we have four possible analyses of $N_{1} N_{2} V_{1} V_{2}$ into NVO assuming no noun is both subject and obof the same verb. However, given two embeddings, three nouns must be assigned each of three verbs as subject and three nouns must be assigned to each of three verbs as object. Still assuming no noun may be assigned as both subject and object of the same verb, we have 18 possible analyses of the double-embedded case (if the final verb is intransitive, there are two possible analyses for the single embedding and 12 for two embeddings). Put another way, in a single embedding the choice of either noun as subject determines the other noun as object. This is not true for two embeddings, where the choice of any three nouns as subject leaves two nouns which may serve as objects.

3. Sentence 7 is excluded from the analysis here and subsequently. The sentence was badly conceived (as the reader can determine for himself by reference to page 000 ). Ss were virtually unanimous in their indignant assertions that the sentence was "meaningless"even those Ss who eventually succeeded in reporting the correct relationships among the sentence elements. In fact, the sentence is best described as a non-sequitur. Exclusion of Sentence 7 does not substantially alter the results.

(Accepted for publication, March 8, 1967.) 